\title{
Light Transport Analysis for 3D Photography
}

\author{
Kiriakos N. Kutulakos \\ Department of Computer Science \\ University of Toronto \\ Toronto, Canada \\ kyros@cs.toronto.edu
}

\begin{abstract}
While $3 D$ photography research has enjoyed tremendous success in recent years, many everyday objects and materials are still difficult or impossible to capture in $3 D$. An important stumbling block is that typical algorithms do not consider the effects of light transport, i.e., the sequence of bounces, refractions and scattering events that may occur when light interacts with an object. This puts objects with transparent materials or highly-reflective surfaces (clear plastic, crystal, liquids, polished metal, etc.) outside the reach of current $3 D$ scanning techniques. To overcome these limitations, we have been investigating algorithms that explicitly analyze the light transport process caused by such objects [1-3]. These algorithms rely on $2 D$ photos taken from multiple views and reconstruct the individual 3 D path(s) that light must have traced in order to reach each pixel. Despite the apparent intractability of this endeavor, our results suggest that reasoning about light transport can produce rich descriptions of surface geometry for objects with complex optical properties.
\end{abstract}

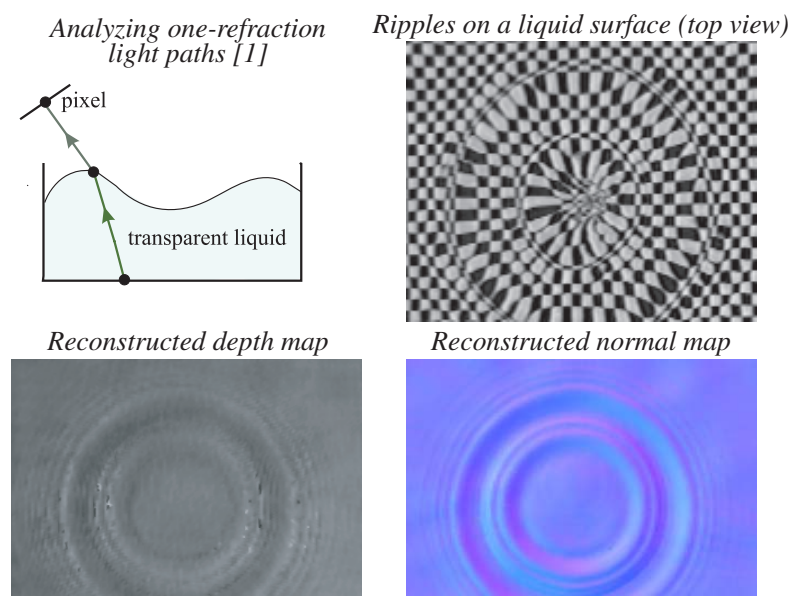

The support of the Natural Sciences and Engineering Research Council of Canada under the RGPIN program, of the National Science Foundation under Grant No. IIS-0413198, and of the Alfred P. Sloan Foundation are gratefully acknowledged.
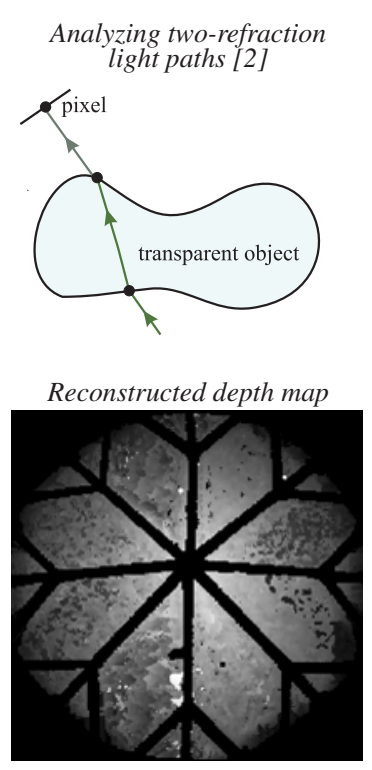

Analyzing \& separating the contribution of one-bounce (red) \& multiple-bounce light paths [3]
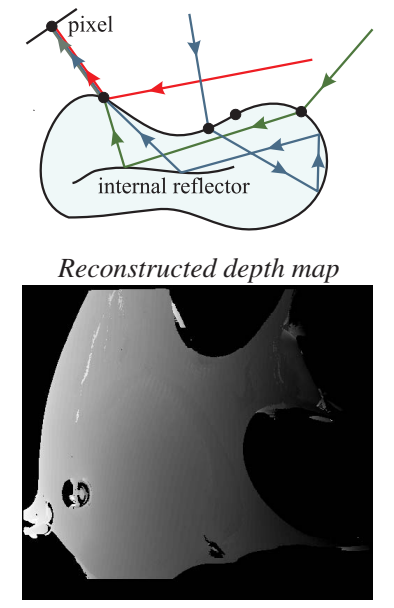

Diamond-shaped object (face-on view)
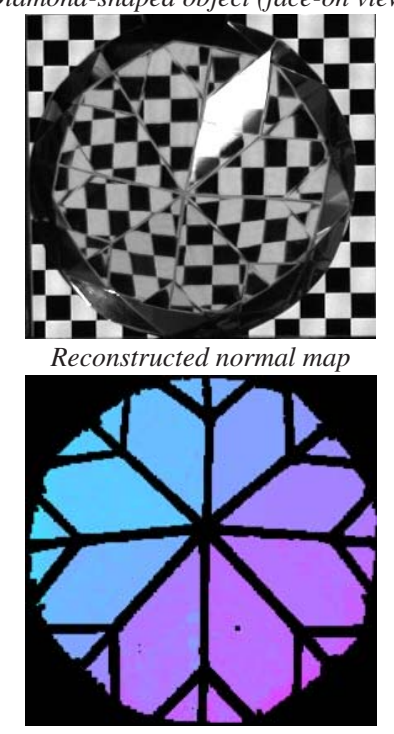

Crystal ornament $w /$ painted interior
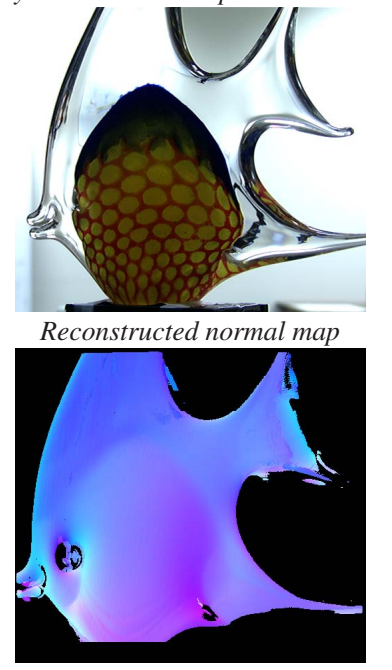

\section{References}

[1] N. Morris and K. N. Kutulakos, "Dynamic refraction stereo," Proc. ICCV'05, pp. 1573-1580.

[2] K. N. Kutulakos and E. Steger, "A theory of and refractive and specular 3d shape by light-path triangulation," Proc. 10th ICCV'05, pp. $1448-1455$.

[3] www.cs.toronto.edu/ ${ }^{k y r o s / r e s e a r c h / s c a t t e r-t r a c e / ~}$ 